\title{
Precision nanoscale domain engineering of lithium niobate via UV laser induced inhibition of poling
}

\author{
C. L. Sones, ${ }^{1}$ A. C. Muir, ${ }^{1, a)}$ Y. J. Ying, ${ }^{1}$ S. Mailis, ${ }^{1}$ R. W. Eason, ${ }^{1}$ T. Jungk, ${ }^{2}$ Á. Hoffmann, ${ }^{2}$ \\ and E. Soergel ${ }^{2}$ \\ ${ }^{1}$ Optoelectronics Research Centre, University of Southampton, Highfield, Southampton, SO17 1BJ, \\ United Kingdom \\ ${ }^{2}$ Institute of Physics, University of Bonn, Wegelerstrasse 8, 53115 Bonn, Germany
}

(Received 10 December 2007; accepted 31 January 2008; published online 20 February 2008)

\begin{abstract}
Continuous wave ultraviolet laser irradiation at $\lambda=244 \mathrm{~nm}$ on the $+z$ face of undoped and $\mathrm{MgO}$ doped congruent lithium niobate single crystals has been observed to inhibit ferroelectric domain inversion. The inhibition occurs directly beneath the illuminated regions, in a depth greater than $100 \mathrm{~nm}$ during subsequent electric field poling of the crystal. Domain inhibition was confirmed by both differential domain etching and piezoresponse force microscopy. This effect allows the formation of arbitrarily shaped domains in lithium niobate and forms the basis of a high spatial resolution microstructuring approach when followed by chemical etching. (C) 2008 American Institute of Physics. [DOI: 10.1063/1.2884185]
\end{abstract}

Domain engineering ${ }^{1,2}$ of lithium niobate $(\mathrm{LN})$ is a subject of extensive research and a simple, cheap, and robust method of fabrication of well-defined periodic domaininverted structures on submicron scales is highly desirable. Spatial domain engineering is used for many optical processes in bulk crystals and waveguides and can also allow for the creation of both freestanding ${ }^{3}$ and surface relief structures ${ }^{4}$ through the differential etching characteristics of the polar $z$ faces of the crystal. If achievable on the submicron scale, surface structuring through differential etching will allow the implementation of a range of interesting applications such as tunable photonic crystals, ridge waveguide lasers, and multifunctional micromachines.

Previous work has shown that ultraviolet (UV) and visible laser light can either directly invert ${ }^{5}$ or assist the domain inversion process in LN. ${ }^{6-9}$ In this paper, however, a different effect is presented whereby illumination of the $+z$ face with UV light at $\lambda=244 \mathrm{~nm}$ (with photon energy greater than the LN band gap) inhibits domain inversion in illuminated areas during subsequent electric field poling (EFP). Of major importance, the inhibited domains are not restricted in their shape or alignment with the crystal $x$ or $y$ axes, hence, arbitrarily shaped domains can be formed. Some initial results of this effect and its applicability in the creation of micro/nano structures in $\mathrm{LN}$ are presented.

A beam from a frequency-doubled Ar-ion laser was focused to a spot size of $\sim 2.5 \mu \mathrm{m}$ on the $+z$ or $-z$ face of either an undoped congruent or $5 \mathrm{~mol} \% \mathrm{MgO}$-doped $\mathrm{LN}$ crystal. Positioning and exposure control of the crystal was achieved by a computer-controlled, three-axis stage system coupled with a mechanical shutter.

For dynamic exposures, sets of parallel lines were drawn on the $z$ faces of the crystals along the crystallographic $x$ or $y$ directions by moving the stages at speeds ranging from 0.05 to $0.3 \mathrm{~mm} \mathrm{~s}^{-1}$. For static exposures, arrays of illuminated spots with identical exposure times, ranging from a few milliseconds to a few tens of seconds, were formed. The

\footnotetext{
${ }^{\text {a) }}$ Author to whom correspondence should be addressed. Electronic mail:
} acm@orc.soton.ac.uk. separation between the edges of adjacent illuminated spots in the arrays varied from 0 to $6 \mu \mathrm{m}$ which permitted us to verify if any proximity effect existed such as that observed in pulsed laser direct poling ${ }^{5}$ where the closest approach observed there was of order $\sim 2 \mu \mathrm{m}$, irrespective of illuminated pattern resolution. For both types of exposures, the power was varied between $20-28 \mathrm{~mW}$.

A two-beam interference scheme that allowed the formation of periodic structures over larger areas of the crystal via single exposures was also investigated. The incident interference fringe pattern had a period of $700 \mathrm{~nm}$ and extended over an area of around $2000 \mu \mathrm{m}^{2}$. As with previous experiments, several different exposure times, ranging from tens of milliseconds to tens of seconds, and a range of incident powers were tried.

Each set of illumination conditions was duplicated for both $+z$ and $-z$ face illuminations, to allow a direct comparison of the resultant effects on each of these polar faces. The samples were then poled using the EFP setup described in Ref. 6. The voltage was ramped at $2 \mathrm{kV} / \mathrm{min}$ to a value of $\sim 10.1 \mathrm{kV}$, corresponding to an electric field of $20.2 \mathrm{kV} / \mathrm{mm}$ across the $0.5 \mathrm{~mm}$ thick sample. This value of the applied electric field ensures that domain inversion occurs slowly, which is desirable since the kinetics of the domain wall motion is seen to influence greatly the shape and quality of the resultant structures.

Etching of the poled crystal in aqueous HF acid solution then allows visualization of the formed domain structures through the different etch rates of the two $z$ faces. ${ }^{10}$ Piezoresponse force microscopy ${ }^{11}$ (PFM) was also used to verify the domain nature of these structures. Lastly, the samples were imaged with a scanning electron microscope (SEM).

Figures 1 and 2 show SEM images of the structures produced by illumination of the single focussed UV beam on the $+z$ face followed by EFP and etching. The images show smooth, continuous unetched sections which correspond to the UV illuminated area of the surface. The etched background is the newly domain inverted area which presents a $-z$ face to the acid and hence etches. Further observation of the SEM images shows that the edges of the structures are 


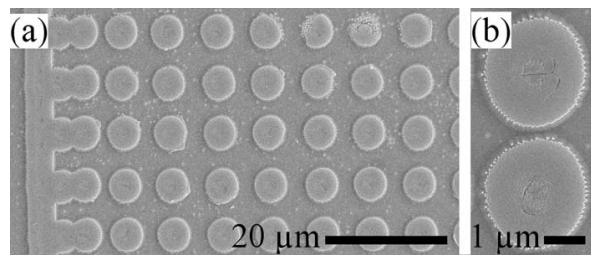

FIG. 1. (a) SEM image of a poling inhibited dot array. Scale bar $20 \mu \mathrm{m}$. (b) SEM image of two closely spaced poling inhibited dots.

slightly jagged, possibly due to the Gaussian intensity profile of the illuminating beam.

Interestingly, exposures on the $-z$ face, done for the purpose of comparison, were not seen to inhibit domain inversion. On the contrary, domain inversion was seen to initiate in the illuminated regions and was then followed by domain propagation through the crystal in a random and disorderly fashion. Hence, all the results discussed in this letter correspond to $+z$ face illuminations that result in domain inhibition.

Arrays of dots, such as the ones shown in Fig. 1(a), which are produced by static exposures, can have arbitrary dot separations and no interaction between dots was observed even when the separation between them was less than one micron, Fig 1(b). When illuminated areas overlapped, the regions of inhibited domain inversion merged. This can be seen on the left hand side of Fig. 1(a), where stage backlash distorted the array by reducing the dot spacing. Additional UV exposure of a specific area was not observed to produce any further effect. This can be seen in Fig. 2(a) where a cross-hatched pattern is shown as a result of sequential line scans along the $x$ and $y$ crystallographic axes. A higher magnification SEM image that shows the quality of the overlapped exposure is shown in Fig. 2(b). The sample was tilted by $45^{\circ}$ during the SEM scan which allows the observation of the quality of the sidewall of the poling inhibited domain. The SEM images shown in Fig. 2 were obtained after prolonged etching $(39 \mathrm{~h})$. As a result, high aspect ratio structures were produced underlining the potential of this method for surface microstructuring.

The potential of this technique to form precisely positioned structures without proximity restrictions demonstrates the practicality of this technique in the implementation of devices such as couplers, $y$ junctions, ring microresonators, and photonic crystals. The width of the area over which inhibition occurs is seen to be dependent upon both the incident power and the dwell time/scanning velocity of the beam with larger domains formed with longer dwell or lower scan speeds. This functionality reiterates the capability of this technique.

The quality of the poling inhibited regions was observed to be dependant upon the domain wall kinetics during the

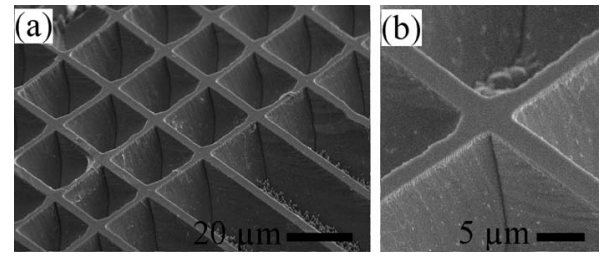

FIG. 2. (a) SEM image of poling inhibited hatched lines after $39 \mathrm{~h}$ of HF etching. Sample tilt $45^{\circ}$. (b) High magnification SEM image of a crossing point in (a).

FIG. 4. SEM image of $+z$ face after interferometric exposure, EFP and brief etching.

Whloaded 10 Nov 2009 to 152.78.208.72. Redistribution subject to AIP license or copyright; see http://apl.aip.org/apl/copyright.jsp

FIG. 3. PFM image of poling inhibited dot array. White $=+z$ face, black $=-z$ face as indicated. White area to left of image is an unpoled bulk domain.

EFP step. It was seen that when the wall movement was smooth and reasonably slow, the inhibition process produced continuous domains. In congruent crystals when the wall movement can be fast, expanding in a "jerky" manner, 1,12 the poling inhibited regions appeared fragmented or did not exist at all in areas corresponding to fast domain wall motion. In the $\mathrm{MgO}$ doped crystals where EFP is known to be slower $^{13,14}$ and smoother, the inhibited domains appeared to be smooth and continuous everywhere.

In order to investigate the stability of the inhibited domains the crystal was thermally annealed for $1 \mathrm{~h}$ at $215^{\circ} \mathrm{C}$ after the EFP step of the process. SEM investigation of the HF etched annealed crystal showed that the poling inhibited domains survived the brief annealing process without any significant change apart perhaps from the fact that after annealing there seems to be a slight improvement on the quality of the edges.

The domain orientation of the inhibited domains was further investigated by PFM which confirmed the observations deduced from chemical etching. Figure 3 shows a PFM image of an area of the crystal that carries a set of static spot illuminations and has also been partially poled (only a fraction of the area was domain inverted). Full contrast is associated with opposite ferroelectric domains where $a+z$ face appears white (left section of the image) while $a-z$ face appears black (right larger section of the image). The dots which appear white in a black background of the PFM image correspond to the UV exposed areas which have maintained their original domain orientation $(+z)$ while the surrounding area has been inverted $(-z)$ and appears black. The depth sensitivity of the PFM is $\sim 100 \mathrm{~nm}$ (Ref. 15) which gives the lower limit to the depth of the inhibited domains. Preliminary investigations of the etched $y$ face indicated that the depth of the domain inhibited area was in the range of $0.5-1 \mu \mathrm{m}$, however a systematic investigation will be presented in a future communication.

Interferometric exposures were at much lower laser intensity than single beam exposures due to the larger spot size. Exposure of the $-z$ face, followed by brief etching in $\mathrm{HF}$, resulted in no topographical contrast. Exposure of the $+z$ face followed by bulk EFP and etching produced a periodic relief structure which is shown in the SEM image of Fig. 4.

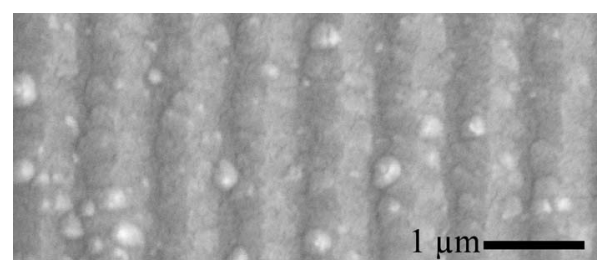


The SEM image shown in Fig. 4 is qualitatively different to the ones produced at higher intensity. We believe that the surface relief pattern shown in Fig. 4 as revealed by chemical etching is a single domain structure which has originated from a periodic poling inhibited structure. Due to the low intensities used in this experiment the initial poling inhibited areas are expected to be very narrow and shallow hence easily removed by the acid via side etching. However, by using higher UV laser intensities it should be possible to obtain deeper periodic domain structures at the highly desired submicron scale.

We believe that the inhibition of the domain reversal is due to redistributed charges under the crystal surface which increases the coercive field locally. UV illumination leads to photoexcitation of charges and heating of the crystal. ${ }^{16} \mathrm{~A}$ pyroelectric field is formed which drives electrons into the bulk of the crystal and holes toward the surface, producing a short range dipolar electric field distribution, which increases the coercive field locally. When a domain nucleates on the $-z$ face and propagates toward $+z$ face, inversion will not occur in this region where the coercive field is increased and domain propagation will be impeded, leaving $a+z$ surface domain island on $a-z$ background. This mechanism has some similarities to that described by Dierolf and Sandmann ${ }^{7}$ for confocal light-assisted poling. However, in our case the driving field for the electrons is created by local heating of the crystal and not an external voltage and is opposite in direction to that of Dierolf and Sandmann, ${ }^{7}$ leading to domain inhibition rather than domain inversion. Also, the mechanism we describe is latent and does not require application of the external field during illumination as in their case.

As a method of surface structuring UV poling inhibition followed by etching is simple, inexpensive, and flexible to implement. The shape/size and the quality of the fabricated structures is determined by the incident exposure conditions, in combination with the subsequent EFP parameters and the annealing steps, respectively. Additionally, as the crystallographic symmetry is not observed to impose limitations on the orientations of the created structures, any desired shapes can be achieved. Also the single domain nature of these structures provides the necessary requirement for implementation of efficient nonlinear, piezoelectric, and other domainorientation-sensitive devices.
In conclusion, exposure of the $+z$ surface of congruent LN single crystals via UV laser radiation at $\lambda=244 \mathrm{~nm}$ is shown to inhibit domain inversion locally during subsequent EFP and, hence, results in the creation of UV illumination controlled two-dimensional surface structures. Domain inhibition was further verified by PFM and differential wet etching. UV light-induced poling inhibition is attributed to the redistribution of photoexcited charges in the electric field created when the laser heats the crystal, thus, producing a space-charge field which repels the propagating bulk domains during the subsequent EFP step. Finally, this effect, in conjunction with wet etching, was demonstrated to provide an easy alternative method for fabricating useful domain and surface relief structures.

The authors are grateful to the Engineering and Physical Sciences Research Council, UK, and the EU for research funding under Grant No. EP/C515668 and STREP 3DDEMO respectively.

${ }^{1}$ V. Y. Shur, Ferroelectrics 340, 3 (2006).

${ }^{2}$ B. J. Rodriguez, R. J. Nemanich, A. Kingon, A. Gruverman, S. V. Kalinin, K. Terabe, X. Y. Lui, and K. Kitamura, Appl. Phys. Lett. 86, 012906 (2005).

${ }^{3}$ C. Sones, S. Mailis, V. Apostolopoulos, I. E. Barry, C. Gawith, P. G. R. Smith, and R. W. Eason, J. Micromech. Microeng. 12, 53 (2002).

${ }^{4}$ S. Mailis, G. W. Ross, L. Reekie, J. A. Abernethy, and R. W. Eason, Electron. Lett. 36, 1801 (2000).

${ }^{5}$ I. T. Wellington, C. E. Valdivia, T. J. Sono, C. L. Sones, S. Mailis, and R. W. Eason, Appl. Surf. Sci. 253, 4215 (2007).

${ }^{6}$ C. L. Sones, M. C. Wengler, C. E. Valdivia, S. Mailis, R. W. Eason, and K. Buse, Appl. Phys. Lett. 86, 212901 (2005).

${ }^{7}$ V. Dierolf and C. Sandmann, Appl. Phys. Lett. 84, 3987 (2004).

${ }^{8}$ M. Müller, E. Soergel, and K. Buse, Appl. Phys. Lett. 83, 1824 (2003).

${ }^{9}$ M. C. Wengler, U. Heinemeyer, E. Soergel, and K. Buse, J. Appl. Phys. 98, 064104 (2005).

${ }^{10}$ C. L. Sones, S. Mailis, W. S. Brocklesby, R. W. Eason, and J. R. Owen, J. Mater. Chem. 12, 295 (2005).

${ }^{11}$ T. Jungk, Á. Hoffmann, and E. Soergel, Appl. Phys. Lett. 89, 163507 (2006).

${ }^{12}$ V. Gopalan, Q. X. Jia, and T. E. Mitchell, Appl. Phys. Lett. 75, 2482 (1999).

${ }^{13}$ A. Kuroda, S. Kurimura, and Y. Uesu, Appl. Phys. Lett. 69, 1565 (1996).

${ }^{14}$ K. Nakamura, J. Kurz, K. Parameswaran, and M. M. Fejer, J. Appl. Phys. 91, 4528 (2002).

${ }^{15}$ T. Jungk, Á. Hoffmann, and E. Soergel, New J. Phys. 10, 013019 (2008).

${ }^{16}$ A. C. Muir, G. J. Daniell, C. P. Please, I. T. Wellington, S. Mailis, and R. W. Eason, Appl. Phys. A: Mater. Sci. Process. 83, 389 (2006). 